\title{
Aspectos fisiológicos do estresse: uma revisão narrativa
}

\author{
Physiological aspects of stress: a narrative review \\ Aspectos fisiológicos del estrés: una revisión narrativa
}

Recebido: 19/11/2021 | Revisado: 23/11/2021 | Aceito: 13/12/2021 | Publicado: 21/12/2021

\author{
Sérgio Ricardo Boff \\ ORCID: https://orcid.org/0000-0002-7675-5755 \\ Universidade Estadual Paulista “Júlio de Mesquita Filho", Brasil \\ Centro Universitário Max Planck, Brasil \\ E-mail: sergioricardoboff@gmail.com \\ Alexandre Gabarra Oliveira \\ ORCID: https://orcid.org/0000-0002-6620-5477 \\ Universidade Estadual Paulista "Júlio de Mesquita Filho", Brasil \\ E-mail: alexandre.gabarra@unesp.br
}

\begin{abstract}
Resumo
Qualquer estímulo seja intrínseco ou extrínseco, desencadeia uma resposta biológica que remete ao estresse. O estresse é um elemento característico do mundo natural, que opera em quase todos os sistemas biológicos, e em cada situação que distancie os sistemas vivos de uma condição fisiológica constante. As respostas fisiológicas podem ser usadas como indicadores de estresse, podendo ser respostas generalizadas a um estímulo, mediadas pelo sistema nervoso simpático e eixo hipotálamo-hipófise-adrenal pela ação de hormônios, como a adrenalina e corticosteroides. Alguns estímulos ativam um sistema mais do que outro, condição que pode ocorrer devido aos papéis específicos do estímulo sobre os sistemas, sendo sobrepostos aos seus papéis generalizados. A amplitude da resposta hormonal pode se correlacionar com a intensidade do estímulo, e qualquer mudança indica que o corpo está respondendo. Com isso, este artigo tem como objetivo analisar a ação fisiológica do organismo frente do estresse. Para tanto, foi realizada uma revisão bibliográfica em que foi utilizada publicações que abordassem o tema nas bases de dados MEDLINE® (PubMed®) e Scientific Electronic Library Online (SciELO) entre os anos de 1983 e 2020. Conclusão: O organismo humano é afetado de modo diverso pelo evento estressor, os impactos dos estressores exercem um efeito estimulante, originando reações comportamentais e fisiológicas usadas para enfrentar estes eventos, uma vez que a resposta adaptativa fundamental à sobrevivência. Existem grandes diferenças individuais em lidar com o estresse, porém, os mecanismos que fundamentam como respondemos suportam as funções fisiológicas, mantendo a homeostase contribuindo para a adaptação.
\end{abstract}

Palavras-chave: Alostase; Estresse; Homeostase; Estresse fisiológico; Estresse psicológico.

\begin{abstract}
Any stimulus, whether intrinsic or extrinsic, triggers a biological response that refers to stress. Stress is a characteristic element of the natural world, operating in almost all biological systems, and in every situation that distances living systems from a constant physiological condition. Physiological responses can be used as stress indicators, and may be generalized responses to a stimulus, mediated by the sympathetic nervous system and the hypothalamic-pituitary-adrenal axis through the action of hormones such as adrenaline and corticosteroids. Some stimuli activate one system more than another, a condition that can occur due to the specific roles of the stimulus on the systems, being superimposed on their generalized roles. The amplitude of the hormonal response can correlate with the intensity of the stimulus, and any change indicates that the body is responding. Thus, this article aims to verify the physiological behavior of the body in the face of stress. For that, a bibliographic review was carried out in which publications that addressed the subject were used in the MEDLINE® (PubMed®) and Scientific Electronic Library Online (SciELO) databases between the years 1983 and 2020. Conclusion: The human body is affected in different ways by the stressful event, the impacts of stressors exert a stimulating effect, giving rise to behavioral and physiological reactions used to face these events, since the adaptive response is fundamental to survival. There are great individual differences in dealing with stress, however, the mechanisms underlying how we respond support physiological functions, maintaining homeostasis and contributing to adaptation.
\end{abstract}

Keywords: Allostasis; Homeostasis; Stress; Stress Physiological; Stress psychological.

\section{Resumen}

Cualquier estímulo, ya sea intrínseco o extrínseco, desencadena una respuesta biológica que se refiere al estrés. El estrés es un elemento característico del mundo natural, que opera en casi todos los sistemas biológicos y en todas las situaciones que alejan a los sistemas vivos de una condición fisiológica constante. Las respuestas fisiológicas pueden utilizarse como indicadores de estrés y pueden ser respuestas generalizadas a un estímulo, mediadas por el sistema 
nervioso simpático y el eje hipotalámico-pituitario-adrenal a través de la acción de hormonas como la adrenalina y los corticosteroides. Algunos estímulos activan un sistema más que otro, condición que puede ocurrir debido a los roles específicos del estímulo sobre los sistemas, sobreponiéndose a sus roles generalizados. La amplitud de la respuesta hormonal puede correlacionarse con la intensidad del estímulo y cualquier cambio indica que el cuerpo está respondiendo. Así, este artículo tiene como objetivo analizar la acción fisiológica del organismo frente al estrés. Para ello, se realizó una revisión de la literatura utilizando publicaciones que abordaron el tema en las bases de datos MEDLINE® (PubMed®) y Scientific Electronic Library Online (SciELO) entre 1983 y 2020. Conclusión: El organismo humano se ve afectado de diferentes formas por el evento estresante, los impactos de los estresores ejercen un efecto estimulante, dando lugar a reacciones conductuales y fisiológicas utilizadas para afrontar estos eventos, ya que la respuesta adaptativa es fundamental para la supervivencia. Existen grandes diferencias individuales al lidiar con el estrés, sin embargo, los mecanismos subyacentes a cómo respondemos apoyan las funciones fisiológicas, mantienen la homeostasis y contribuyen a la adaptación.

Palabras clave: Alostasis; Estrés; Homeostasis; Estrés fisiológico; Estrés psicológico.

\section{Introdução}

O homem sofre com exposição a diferentes estímulos como o trabalho, medo, exposição ao calor e frio, fome, sede, doença. A vida moderna leva o indivíduo a necessidade de mudanças expondo pessoas a situações geradoras de conflito, ansiedade, angústia e desestabilização emocional. Como consequência, o estresse é resultante dos esforços do indivíduo em adaptar-se à situação estressora. O estresse nem sempre é razão de esgotamento emocional e físico, e sim, um instrumento natural de defesa da pessoa (Aroldi-Favassa, 2005).

O estresse mental ou emocional é uma das maiores adversidades da sociedade moderna. Em um cenário de estresse, o organismo humano redistribui suas fontes de energia antecipando uma ofensiva próxima. Esse mecanismo é útil, mas se realmente houver risco, entretanto, se esse estado prosseguir por muito tempo, o prejuízo será inevitável (Loures et al., 2002). Esta condição vai ao encontro do proposto por Selye (1936), que descreve o estresse como um reflexo do esgotamento sofrido pelo organismo frente aos desafios da ambientação, situação em que os organismos vivos demonstram mudanças em resposta a estressores, e caso medidas de controle não sejam tomadas pode-se levar a deterioração física e psicológica.

O estresse é um ponto de referência importante em estudos de saúde, e está relacionado ao estado geral de saúde de um indivíduo e as diferentes doenças, incluindo transtornos mentais, câncer, doenças cardiovasculares, abuso de drogas, doenças crônicas, entre outras. Sendo o estresse um sintoma transcultural para muitos tipos diferentes de problemas de saúde, sua compreensão em diferentes grupos sociodemográficos, culturais e sociais, pode ajudar a prevenir problemas relacionados ao próprio estresse e os principais problemas de saúde em todo o mundo (Vallejo, 2018).

A conceituação de estresse tem sido abundantemente utilizada nos dias atuais, tendo relação com a ideia de esforço, tensão, tornou-se senso comum, e a cultura popular há muito vincula o estresse com o desdobramento de doenças, validado por inúmeros estudos epidemiológicos e experimentais, que ratificam uma ligação entre o estresse e a manifestação em curso de muitas doenças, desde simples infecções virais até úlceras gástricas e neoplasias (Loures et al., 2002). Portanto, a definição do estresse leva a um amplo debate quando se busca compreender o assunto, pois, aborda aspectos que vão desde a fisiologia até às ciências sociais, e está estreitamente associado à possibilidade de adaptação e à mudança.

Pode ser visto como um conteúdo multidisciplinar que hoje apresenta a abrangência de visões do estresse, pois é compreendido como evento psicossocial que reflete sobre a atividade neurofisiológica, e disparado quando acontece a sensação de uma ameaça legitima ou fictícia, quando manifesta a habilidade de abalar a plenitude física e/ou mental de um sujeito (Santos, 2014).

Já, para as ciências sociais o interesse manifesta-se associado ao entendimento de que o ambiente sócio histórico acarreta graus de manifestação e aptidão adaptativa variados, deste modo, o estresse é idealizado por meio da soma de fatores sociais que modificam a ambientação do indivíduo, visto que é a provável inabilidade de ajuste com as necessidades 
adaptativas do meio que converte-se em impactos danosos no tocante a saúde, e não simples resposta espontânea aos estímulos estressógenos (Faro, 2013).

Na psicologia, atualmente, partilham-se alguns princípios do estresse com as ciências sociais e, também, reitera-se o efeito sobre a atividade biológica, mas seu prisma explicativo parte principalmente do indivíduo para o meio. Diante disso, um conceito básico para a psicologia é o estresse como uma associação íntima entre o indivíduo e o ambiente, tornando- se fundamental interpretar qualquer artifício psicológico e social que intervenha este contato (Faro, 2013).

Sendo assim, o objetivo desta revisão foi verificar a resposta fisiológica do indivíduo diante o estresse, para o melhor entendimento deste mecanismo, e apresentamos ao longo do texto a concepção clássica de resposta de estresse, a ideia de homeostase e a teoria da alostase, e subsequentemente, os mecanismos de ajustes fisiológicos regulatórios responsáveis pelas respostas fisiológicas frente a condições de instabilidade.

\section{Metodologia}

Este trabalho trata-se de uma revisão crítica do tipo narrativa da literatura para reconhecer o estado da arte a respeito do tema estresse, sob a lógica da fisiologia. Este tipo de revisão é uma forma não sistematizada de revisar a literatura, apresenta um assunto de forma mais aberta; e raramente parte de uma questão particular bem estabelecida, é baseada em um processo mais simplificado, e a questão de pesquisa pode ser mais abrangente, ou pouco exclusiva, além de abordar um tema de forma livre, não há imposição aos autores para que informem de maneira detalhada os procedimentos ou critérios usados para a escolha e avaliação das referências incluídas, pois a forma de seleção é variável e arbitrária (Casarin et al, 2020).

Para a elaboração deste estudo, foram utilizadas publicações que abordassem o tema, após análise e seleção de artigos encontrados pela de busca nas bases de dados MEDLINE® (PubMed®) e Scientific Electronic Library Online (SciELO). Para tal, utilizou-se os Descritores em Ciências da Saúde (DeCS) Allostasis, Homeostasis, Stress, Stress Physiological, Stress Psychological, o operador booleano AND e suas correspondentes em português. O critério de inclusão para a busca dos estudos foi: artigos publicados sobre o tema em periódicos no período de 1983 a 2020, não houve restrição de idioma. Os critérios de exclusão: artigos que não possuíam relação com a questão principal do estudo, artigo anterior ao período estipulado, monografias, teses e dissertações.

\section{Resultados e Discussão}

\section{Fisiologia do Estresse: homeostase e alostase}

As primeiras evidências práticas que levaram a posterior descrição de um modelo experimental do estresse foram conduzidas por Hans Selye. O trabalho de Selye (1936) converteu-se em uma referência na história do estudo do estresse ao examinar as ações fisiológicas dos hormônios sexuais, após injetar em ratos amostras hormonais de outros animais, e, submetêlos ao frio, injúria tecidual, excesso de exercícios e intoxicações. Os achados obtidos através de necroscopia incluíram hipertrofia das glândulas adrenais, presença de úlceras gástricas e atrofia de órgãos linfoides como: timo, baço e linfonodos. Como tais achados tornam-se independentes do estímulo aplicado, Seyle (1959) constatou que correspondiam a uma resposta orgânica ao dano, baseado neste conjunto de respostas fisiológicas, propondo que as reações, ou respostas, ao estresse seja denominada Síndrome de Adaptação Geral, a qual possui três estágios: reação de alarme, fase de resistência e fase de exaustão. Cada um destes estágios envolve respostas fisiológicas e comportamentais, e caso as medidas de controle não sejam tomadas pode-se levar a deterioração física e psicológica.

A reação de alarme consiste no organismo em reconhecer a situação estressora e mobilizá-lo para o enfrentamento da mesma, tal feedback acelerado é mediado, especialmente, pelo estímulo do sistema nervoso autônomo simpático o qual proporciona a liberação de neurotransmissores em vários órgãos-alvo, e além disso ativa a medula das glândulas adrenais a 
disponibilizar os hormônios catecolaminérgicos, adrenalina e noradrenalina, ampliando ainda mais a ativação neural (AraldiFavassa, 2005; Sousa, 2015).

Na persistência do estímulo os instrumentos destinados ao enfrentamento estão conservados, e as respostas são, habitualmente, adaptativas, o que amplia as possibilidades de sobrevivência do indivíduo, referindo-se esta a fase de resistência. Com a finalidade de resgatar a homeostase, as respostas fisiológicas e comportamentais são conservadas e mediadas, especialmente pelo cortisol. Neste momento a efetividade das respostas alcança sua condição máxima e o indivíduo exibe sua melhor performance física e cognitiva, e desta maneira exibe total possibilidade para anular o agente estressor (Araldi-Favassa, 2005; Sousa, 2015).

No momento em que este ajuste não acontece o organismo falha em anular o agente estressor e segue respondendo de forma crônica, e consequentemente, muitas funções fisiológicas fundamentais do organismo podem ser afetadas, particularmente: metabolismo, crescimento, reprodução e imunidade, caracterizando assim a fase de exaustão (Araldi-Favassa, 2005; Sousa, 2015).

O uso deste conceito demanda uma compreensão dos mecanismos fisiológicos que geram uma resposta integrada de enfrentamento a situações adversas, que remetemos ao conceito clássico de homeostase (Souza, 2015).

O conceito de homeostase é um pilar central da fisiologia moderna, o termo foi apresentado por Cannon (1929) e se refere à manutenção da estabilidade do meio interno, ou seja, segundo Brito (2017), o conceito de homeostasia de Cannon sugere que o ser vivo, é um sistema aberto com muitas relações com o seu entorno. Nesse sentido, alterações nas circunvizinhanças geram reações nos diferentes sistemas orgânicos, ou os acometem continuamente, de maneira que disfunções internas do sistema são desenvolvidas. Estes distúrbios são usualmente mantidos dentro de uma margem estreita, pois ajustes automáticos dentro do sistema são colocados em atividade, e, consequentemente, significativas mudanças são inibidas e o ambiente interno mantem-se praticamente inalterável.

Cannon (1929) sugere ainda que parâmetros relevantes para a manutenção do ambiente pericelular, por exemplo, pressão arterial, frequência cardíaca e glicemia, embora geralmente permaneçam dentro de certas 'faixas normais' quando o corpo está em repouso, precisariam estar ativamente alterados em outras ocasiões para atender às necessidades teciduais. Dessa forma, o autor alega que a homeostase seria alcançada por meio de o cérebro e os nervos, o coração, os pulmões, os rins e o baço, todos trabalhando cooperativamente, ou seja, certamente não apenas por mecanismos locais de feedback negativo.

Neste sentido, Day (2005) sugere que o conceito de homeostase de Cannon não indica que todos os parâmetros fisiológicos devem ser mantidos constantes em todos os momentos, mas apenas aqueles essenciais para a sobrevivência. Considera-se assim que o corpo opera com base na obtenção de "estabilidade por meio da mudança" como parte integrante do conceito de homeostase.

Ainda sobre do conceito da homeostase, McEwen (2003), sugere que a estabilidade dos sistemas fisiológicos que mantêm a vida, como $\mathrm{pH}$, temperatura corporal, níveis de glicose e tensão de oxigênio devem ser mantidos dentro de uma faixa ideal de acordo com a demanda, ou seja, certos parâmetros fisiológicos parecem ser redefinidos para novos intervalos de acordo com as circunstâncias. Nesse contexto, o estresse é frequentemente definido como uma ameaça real à homeostase, e geralmente se refere a um evento ou sucessão de eventos que causam uma resposta, muitas vezes na forma de "sofrimento", mas também, em alguns casos, referindo-se a um desafio que leva a um sentimento de alegria, como um "bom estresse".

O termo estresse está cheio de ambiguidades, e também é usado para significar o evento (estressor) ou a resposta (resposta ao estresse). É frequentemente usado no sentido negativo de "angústia" e, às vezes, para descrever um estado crônico de desequilíbrio na resposta ao estresse, sendo também considerado como evento, ou eventos, que são interpretados como ameaçadores, e que provocam respostas fisiológicas e comportamentais. A esse respeito, é notável que, nos últimos anos, tenha sido argumentado que o conceito de homeostase deve ser suplementado pelos conceitos de alostase e carga alostática. Um dos 
supostos benefícios dessa mudança é que ela esclarecerá o conceito de estresse (Day, 2005).

O conceito de alostase consiste em alcançar estabilidade por meio da mudança (Sterling, 1988; McEwen, 2003) e, sua relevância para o contexto fisiológico foi reconhecida por Creel et al., (2013) e Schulte (2014). Este processo dá suporte à homeostase, ou seja, aqueles parâmetros fisiológicos essenciais à vida, conforme os ambientes e ou as fases da história de vida mudam, isso significa que os "pontos de ajuste" e outros limites de controle também devem mudar. Trata-se do processo que leva à adaptação ou aclimatação do organismo no curto prazo, e os mediadores da alostase incluem os hormônios do estresse, bem como o sistema nervoso autônomo (SNA), as citocinas pró-inflamatórias, e os hormônios metabólicos (Freire et al., 2020).

A alostase também esclarece a ambiguidade inerente ao termo homeostase e distingue entre os sistemas que são essenciais para a vida (homeostase) e aqueles que mantêm esses sistemas em equilíbrio (alostase). Os sistemas alostáticos permitem que um organismo responda ao seu estado físico, por exemplo, acordado, adormecido, supino, em pé, se exercitando, e lide com ruído, aglomeração, isolamento, fome, temperaturas extremas, perigo físico, estresse psicossocial e microrganismos ou parasitas infecções, possibilitando assim a adaptação de parâmetros fisiológicos a mudanças que alteram a ideia de equilíbrio. Essas flutuações tornam possível para o indivíduo funcionar em resposta a um ambiente social e físico em mudança (McEwen, 2016).

A ideia de carga alostática foi introduzida por McEwen (1993), para se referir ao desgaste que resulta de muito estresse ou do gerenciamento ineficiente de alostase, por exemplo, não desligar a resposta quando não é mais necessária, desta forma a carga alostática, segundo Kudielka (2001), é definida como o custo da exposição crônica a respostas endócrinas ou neurais elevadas ou flutuantes resultantes de desafios crônicos ou repetidos que o indivíduo experimenta como estressantes. Portanto, a carga alostática fornece uma estrutura útil para conceituar o impacto fisiológico multissistêmico do estresse, sustentando seus efeitos na saúde e no bem-estar, e as consequências da sobrecarga alostática incluem muitas doenças comuns da vida moderna (McEwen, 1993).

A carga alostática e sua forma mais grave, a sobrecarga alostática, representam os efeitos cumulativos do estresse fisiológico crônico (Berger et al., 2019; Freire et al., 2020). Sendo assim, as situações que desregulam os mediadores da alostase e podem levar ao desenvolvimento de carga/sobrecarga alostática são: (a) exposição a estressores frequentes que podem determinar um estado de estresse crônico e excitação fisiológica repetida; (b) falta de adaptação a estressores repetidos; (c) incapacidade de interromper a resposta ao estresse após o término de um estressor; (d) resposta alostática insuficiente para lidar com o estressor (Guidi et al., 2020).

O impacto fisiológico multissistêmico do estresse sustentado, bem como seus efeitos na saúde e no bem-estar, estrutura-se na carga alostática, este modelo descreve o custo biológico exigido pela ativação prolongada de marcadores primários no SNA e no sistema hipotálamo-pituitária-adrenocortical. Quando um organismo tenta manter a "alostase", isto é, homeostase fisiológica, em face de desafios ambientais, psicológicos e comportamentais, as respostas cumulativas ao estresse podem ter efeitos prejudiciais em várias funções fisiológicas secundárias, aumentando assim os riscos de morbidade e mortalidade, conceituados como resultados terciários no modelo de carga alostática. O modelo reconhece também, que existe uma grande variação nas consequências fisiológicas e de saúde do estresse crônico em função da interação entre influências genéticas, ambientais e individuais (Gallo, 2014; Berger et al., 2019, Kerr et al., 2020).

Assim, em uma revisão da literatura sobre o tema, McEwen (2003) escreveu que o estresse descreve eventos que ameaçam a fisiologia individual provocando respostas comportamentais como parte de alostase além do imposto pelo ciclo de vida normal. Ele propõe ainda, que o estresse é apenas um tipo de desafio que pode ativar as respostas alostáticas, desta forma, a vida apresenta uma série de desafios; alguns fazem parte do ciclo de vida normal, enquanto alguns podem ser descritos como estressores. Todos esses desafios devem ser enfrentados, ou seja, a homeostase deve ser mantida, e esta manutenção envolve 
algum desgaste (carga alostática) que pode ter um impacto adverso na saúde.

Para Fava et al., (2019) a carga alostática reflete os efeitos cumulativos de experiências estressantes na vida diária, e pode levar a doenças ao longo do tempo, desencadeadas quando há exposição crônica a respostas neurais e fisiológicas sistêmicas flutuantes, ou intensificadas que excedem os recursos de enfrentamento de um indivíduo. Tal situação é referida por Shonkoff (2009) como "estresse tóxico", ou seja, que afeta a arquitetura do cérebro, interfere em demais sistemas orgânicos e leva os sistemas de coordenação de estresse a fixarem limites um tanto mais baixo de potencial de resposta, que podem persistir ao longo da vida, aumentando assim o risco de doenças relacionadas ao estresse e comprometimento cognitivo, isso tudo, segue-se a uma sobrecarga alostática. Em contrapartida, Shonkoff (2009) sugere ainda que o estresse positivo é marcado por incremento mediano e de curto período na frequência cardíaca, pressão arterial, e níveis de hormônio do estresse. A característica inerente do estresse positivo é justamente ser um fator significativo do desenvolvimento saudável, experimentado no ambiente de relações constantes e de suporte que permitem respostas adaptativas, que por sua vez recompõe o sistema de resposta ao estresse ao estado original.

O estresse aceitável é uma situação que torna-se iminentemente perturbador, mas é protegido pelos recursos pessoais e interpessoais do indivíduo, e ocorre dentro de um período limitado de tempo, enquanto, no estresse tóxico a ativação é forte, frequente e/ou prolongada do sistema de resposta ao estresse corporal e na ausência de fatores de amortecimento ou proteção é fácil definir, nesta condição, a sobrecarga alostática como a transição para este estado extremo (Shonkoff, 2009; Fava et al., 2019). Para Fava et al. (2019), carga alostática é o preço da exibição crônica a respostas neurais, ou neuroendócrinas ondulantes ou amplas, decorrentes de desafios ambientais repetidos ou crônicos, aos quais um indivíduo reage como sendo particularmente estressante, e refere-se ao desgaste que resulta de muito estresse, ou do gerenciamento ineficiente da alostase.

\section{Controle da resposta ao estresse}

A resposta a um estímulo estressante é consequência da interligação por meio dos atributos da pessoa e as exigências do meio, isto é, as diferenças entre o meio externo e interno, e o entendimento do indivíduo no que se refere a sua habilidade de resposta a este. Esta resposta apresenta pontos cognitivos, comportamentais e fisiológicos, aspirando possibilitar maior compreensão do cenário e de suas demandas, desse modo como um processamento mais hábil da informação à disposição, proporcionando a procura de respostas, e buscando condutas apropriadas, além de estimulando o organismo a fim de operar de modo rápido e intenso (Margis, 2003). Frente a estímulos estressores a resposta possui características específicas, modificando conforme o seu tipo, intensidade e duração, e também concomitante aos aspectos individuais, sendo que as experiências anteriormente vivenciadas pelo indivíduo igualmente têm considerável interferência em respostas fisiológicas a estímulos estressores (De Rose, 2002). Desta maneira, em dose apropriada o estresse é capaz de propiciar ao indivíduo maiores oportunidades para reagir e adotar resoluções, agindo na qualidade de elemento positivo, o qual estimula a conquista de habilidades e superação de obstáculos (Poletto, 2009; Trícoli, 2010). No entanto, no momento em que algum limite é transposto, o estresse consegue oferecer sérios prejuízos à integridade dos indivíduos (Ferreira, 2012).

Respostas adequadas ao estresse alteram processos fisiológicos e comportamentais. Estímulos que geram mudanças e potencialmente ameaçam o equilíbrio, demandam alocação de recursos que podem ser resumidos no aumento do gasto energético objetivando a manutenção da sobrevivência (Angelier, 2013). Os glicocorticoides são um componente importante dessa resposta, mediada principalmente por mecanismos fisiológicos que envolvem os sistemas nervoso e endócrino, no intuito de redistribuir recursos energéticos e restaurar a homeostase após o desafio imediato ter diminuído, (Angelier, 2013).

Atividade combinada de diferentes sistemas centrais de regulação atuando em interação para mobilizar energia para o organismo promove a resposta ao estresse, isto acontece como consequência ou em antecipação a um desafio levando a uma consulta às experiências anteriores para prever a necessidade de mobilização energética, (Myers, 2014). Para este controle, os 
glicocorticóides, especificamente o cortisol no homem, e corticosterona nos roedores, hormônios esteroides sintetizados e liberados pelas glândulas adrenais, em resposta a estímulos fisiológicos e estresse, influenciam o metabolismo celular acarretando a movimentação de substratos energéticos, a fim de que aconteça a elaboração de reações fisiológicas adaptativas defronte os agentes estressores (Pacák, 2001; Ramamoorthy, 2016). Tal resposta é mediada principalmente por sinais neurais originados no núcleo paraventricular do hipotálamo, envolvendo também a glândula pituitária anterior e o córtex da glândula adrenal, conjuntamente denominadas de eixo hipotálamo-pituitária-adrenal (HPA), que em interação respondem a ameaças iminentes ou percebidas que provocam uma resposta ao estresse, culminando principalmente na liberação de glicocorticoides na circulação sistêmica pelas glândulas suprarrenais (Burford, 2017).

Além do eixo HPA, o SNA, através do eixo Sistema Nervoso Simpático - Medula Adrenal, fornece uma resposta mais imediata à exposição ao estressor, provocando mudanças aceleradas nos estados fisiológicos por meio da inervação neural de órgãos terminais, com aumento da secreção de glicocorticoides e catecolaminas (Ulrich-Lai, 2009; Myers, 2014). A respectiva contribuição dos sistemas neuroendócrino e autonômico são sintonizadas de acordo com a modalidade ou intensidade do estressor (Ulrich-Lai, 2009), sendo a resposta neuroendócrina ao estresse um processo temporal altamente regulado, envolvendo a integração de informações sensoriais de múltiplas modalidades (Myers, 2014).

Vale lembrar a importância do sistema renina-angiotensina-aldosterona na modulação da resposta ao estresse uma vez que este sistema é alterado em consequência do estresse. A inervação renal pelo sistema simpático resulta na produção de renina, iniciando uma série de reações culminando com a produção de angiotensina II (Steven, 2018). Eventos de estresse estão relacionados a certa elevação dos níveis de renina e angiotensina II que irá atuar principalmente em receptores do tipo AT1 modulando consideráveis ajustes ao estresse, uma vez que estes receptores estão situados na musculatura lisa vascular, musculatura cardíaca, gânglios simpáticos, na adrenal e na hipófise, desta maneira. Sendo assim, a angiotensina II participa das respostas cardiovasculares ao estresse ao interagir de modo direto com tais receptores vasculares ou cardíacos ora de modo indireto, pelo meio da modulação da atividade simpática, elevando a pressão arterial e a frequência cardíaca. (Reina-Couto et al., 2021; Steven, 2018).

A estimulação dos componentes fundamentais da resposta ao estresse, tem como objetivo aumentar rapidamente a circulação sanguínea e mobilizar substratos energéticos com destino ao sistema nervoso central, tecido muscular e coração (Pacák, 2001; Ranabir, 2011), através de alterações fisiológicas como aumento da frequência e força da contração cardíaca, da pressão arterial, (Liu et al, 2019) aumento da frequência respiratória (Nicolo, 2020), elevação da glicemia por meio da ampliação do catabolismo do glicogênio, uma vez que o cortisol promove a gliconeogênese no fígado (Tanno, 2002; Gaiato et al, 2019) aumento do metabolismo celular e da atividade mental, todas essas alterações permitem um desempenho físico e mental aumentado (Ranabir, 2011; Sousa, 2015).

\section{Cortisol e estresse}

O crescimento nos níveis de estresse, quer seja de motivação psicológica, física ora ambiental, decorre na maior secreção de hormônios glicocorticoides, sendo o cortisol, um hormônio lipídico originário do colesterol, o mais importante, elaborado pelo córtex adrenal humano, é responsável por volta de 95\% de toda a atuação glicocorticoide do organismo (Santos, 2014).

A liberação do cortisol é caracterizada por um padrão circadiano de secreção basal pelas glândulas supra-renais, com os níveis mais altos sendo vistos logo antes do início do ciclo ativo, ou seja, antes do despertar, uma vez que é necessária uma ativação antecipatória para preparar o animal para a fase ativa do dia (diurno para o homem e noturno para animais noturnos, incluindo a maioria das espécies de roedores), seguido por um declínio gradual, atingindo níveis mais baixos durante períodos diários de maior inatividade. A variação circadiana do cortisol também interage com várias outras oscilações biológicas 
importantes, como a atividade diária, a temperatura corporal e a atividade transcricional de muitos genes responsivos aos glicocorticoides, sendo o padrão de secreção altamente individual. Mais especificamente, variações são observadas de acordo com hormônios sexuais, estressores ambientais e mudanças associadas com a idade, enfim, o sistema deve mostrar plasticidade para facilitar a adaptação às novas circunstâncias (Russell, 2015; Lightman, 2020).

A variação circadiana observada no ritmo natural do cortisol é composta de pulsos discretos subjacentes de atividade HPA, um ritmo ultradiano rápido e dinâmico. Este padrão pulsátil de secreção é produzido em uma frequência de aproximadamente 60-90 minutos com um aumento na amplitude de pulso e frequência correspondente ao pico circadiano de secreção, (Russell, 2015) isso foi documentado em todas as espécies de mamíferos estudadas, incluindo humanos (Veldhuis et al., 1989).

Os ritmos circadianos, como exemplificados pelo ciclo sono / vigília, são as manifestações externas de um sistema de temporização interno, em mamíferos, a luz é o sinal mais potente, tendo o Trato Retino-Hipotalâmico, como a principal via, por meio desta, a informação chega ao Sistema Nervoso Central, especificamente no Núcleo Supraquiasmático do hipotálamo anterior, ponto focal desse sistema, sendo o relógio mestre, que orquestra o programa circadiano e funciona como um temporizador, além de ter a capacidade de ser reiniciado por estímulos ambientais (Reppert, 2002).

As entradas do Núcleo Supraquiasmático estimulam o Núcleo Paraventricular do hipotálamo a liberar o Hormônio Liberador de Corticotrofina (CRH) e Vasopressina Arginina, também conhecido como hormônio antidiurético. Esses hormônios atuam na Hipófise anterior, onde ativam as células corticotróficas para secretar o Hormônio Adrenocorticotrófico (ACTH) na circulação geral. Posteriormente, o ACTH atua no córtex adrenal para estimular a síntese e liberação de glicocorticoides. Uma vez liberados pelas glândulas supra-renais para a circulação sanguínea, os glicocorticoides acessam os tecidos-alvo para regular uma série de processos fisiológicos, incluindo metabolismo, função imunológica, crescimento esquelético, função cardiovascular, reprodução e cognição. Devido à sua natureza lipofílica, os glicocorticoides não podem ser pré-sintetizados e armazenados nas glândulas supra-renais, mas devem ser rapidamente sintetizados (usando uma série de reações enzimáticas) após a estimulação com ACTH. Este mecanismo dentro do eixo HPA é equilibrado pelo feedback negativo dos glicocorticoides agindo tanto na hipófise, anterior quanto dentro do hipotálamo para inibir a liberação posterior de ACTH e CRH, respectivamente (Ramamoorthy, 2016; Lightman, 2020).

Além da ação do ritmo circadiano, a resposta ao estresse também leva à ativação do eixo HPA, em que o cortisol é o principal efetor, e do sistema simpato-adreno-medular, que envolve a liberação das catecolaminas, adrenalina e noradrenalina (Pereira, 2012). Regiões límbicas influenciam na ativação do eixo HPA e desempenham funções importantes nas respostas autonômicas ao estresse. Sinais estressores ativam o Sistema Límbico e são direcionados ao hipotálamo, especificamente ao Núcleo Paraventricular, por onde ocorre a ativação do SNA, que por sua vez fornece uma resposta mais imediata à exposição ao estressor por meio de seu braço Simpático, provocando alterações rápidas nos estados fisiológicos por meio da inervação neural de órgãos terminais a medula adrenal. A exposição ao estressor resulta na ativação de neurônios simpáticos da medula espinhal que se projetam para os órgãos terminais, via nervos simpáticos e para as células cromafins da medula adrenal, aumentando os níveis circulantes de adrenalina, via medula adrenal e noradrenalina, via nervos simpáticos (Ulrich-Lai, 2009; Ranabir, 2011; Nicolaides, 2015).

Após aproximadamente 15 minutos do início de um estressor as concentrações de cortisol atingem o pico (Shirtcliff, 2015), e como produto final do eixo HPA encerra a resposta ao estresse agindo principalmente no hipotálamo e na glândula pituitária, formando assim alças de feedback negativo na secreção de CRH e ACTH, respectivamente (Pereira, 2012; Nicolaides, 2015).

O cortisol é uma molécula hidrofóbica, 80 a $90 \%$ do cortisol que está em circulação no sangue e encontra-se combinado a proteínas carreadoras, a globulina ligadora de corticosteroides (CBG), também conhecida como transcortina, 10 a 
$15 \%$ do cortisol está acoplado à albumina, o restante aproximadamente 5\% do cortisol circulante constitui a fração livre, fração, esta que, regula negativamente sua própria secreção ao inibir a liberação de CRH e ACTH (Shirtcliff, 2015). Vale lembrar, que a concentração do cortisol na saliva reflete a fração livre do hormônio no sangue (Silva, 2007), visto que move-se por entre as células acinares a fim de adentrar na saliva mediante difusão passiva (Shirtcliff, 2015). Como um hormônio lipossolúvel, o cortisol atravessa facilmente as membranas celulares, o que permite que ele viaje pelo citosol diretamente para se ligar ao receptor de glicocorticóide intracelular localizado no núcleo da célula onde se dimeriza, se transloca e regula a expressão do gene da maioria das células humanas, especialmente no cérebro, é responsável por encerrar a resposta ao estresse por meio de feedback negativo (Shirtcliff, 2015; Verbeeten, 2018).

Aumento dos níveis cortisol também podem acontecer, independentemente do ritmo circadiano e de fatores estressores (Soares, 2006; Kim, 2009), mas também em resposta ao estresse físico e psicológico (Chernow, 1987), atividade física (Reynoso-Sánchez et al., 2017), esporte de alto rendimento (Farias, 2021), dieta e comportamento alimentar associado a recompensa (Matos, 2021), além do uso de fármacos (Inder, 2012; Maidana, 2013). Com isso, alguns fatores podem ser identificados por interferirem e gerarem diferenças na interpretação dos resultados de dosagens hormonais do eixo HPA (Castro, 2003), com destaque para o sexo, superfície corporal, horas de sono noturno e número de refeições diárias (Castro, 2002). Considera-se também que esta interferência pode ser produzida pela hipertensão arterial, diabetes, síndrome metabólica, síndrome de Cushing, uso exógeno de glicocorticoides e tumores adrenais (Silva, 2007). Além disso, há relação entre hipercortisolemia, depressão, transtornos bipolares, uso de fluoxetina (Alheira, 2005), e também podem ocorrer interferências na interpretação como a diminuição do cortisol produzida pelo uso de drogas como lítio, esteróides sintéticos, cetoconazol, aminoglutetimida e metirapona (Cordás, 2006).

\section{Considerações Finais}

No organismo humano o episódio gerador de estresse, ou estímulo, atinge o indivíduo de maneira variada e a resposta ao estresse é um mecanismo de extrema importância para a adaptação ao ambiente, uma vez que é através desta que nos é permitido o enfrentamento ou a proteção, frente uma situação de perigo.

Os eventos ou agentes estressores exercem um efeito estimulante, acarretando respostas comportamentais e fisiológicas empregadas para enfrentar estes desafios. Desta forma, o estresse nos coloca em alerta preparando a pessoa para uma ação e proporcionando a melhora do desempenho. Porém, quando o estímulo estressor é persistente e crônico, ele deixa de ser saudável, e passa a apresentar efeitos maléficos comprometendo o bem estar, e consequentemente a qualidade de vida

Existem grandes diferenças individuais em lidar com o estresse, os mecanismos que fundamentam como respondemos ao estresse suportam as funções fisiológicas, os quais envolvem a participação de diferentes sistemas na tentativa de manter a homeostase perturbada pelo estímulo ou agente estressor, contribuindo para a adaptação. A manutenção da homeostase é importante e necessária para o funcionamento fisiológico do organismo, pois o desequilíbrio, pode resultar em estados patológicos.

O cenário do presente estudo faz alusão a mecanismos claros relacionados ao estresse de uma forma genérica, sugerimos a realização de novos estudos, associando a condição de estresse a populações específicas, uma vez que, estímulos diferentes e com diferentes intensidades podem proporcionar manifestações adversas.

\section{Referências}

Alheira, F. V., \& Brasil, M. A. A. (2005). O papel dos glicocorticóides na expressão dos sintomas de humor: uma revisão. Revista de Psiquiatria do Rio Grande do Sul, 27 (2), 177-186. https://doi.org/10.1590/S0101-81082005000200008.

Angelier, F., \& Wingfield, J. C. (2013). Importance of the glucocorticoid stress response in a changing world: Theory, hypotheses and perspectives, General and Comparative Endocrinology, 190 (1), 118-128. https://doi.org/ 10.1016/j.ygcen.2013.05.022. 
Araldi-Favassa, C. T., Armiliato, N., \& Kalinine, I. (2005). Aspectos Fisiológicos e Psicológicos do Estresse. Revista de Psicologia da UnC, 2 , (2), 84 - 92.

Berger, M., Taylor, S., Harriss, L., Campbell, S., Thompson, F., Jones, S., Sushames, A., Amminger, G. P., Sarnyai, Z., \& Mcdermott, R. (2019). Hair cortisol, allostatic load, and depressive symptoms in Australian Aboriginal and Torres Strait Islander people. Stress, 22 (3), 312-320. https://doi.org/ 10.1080/10253890.2019.1572745.

Brito, I., \& Haddad, H. (2017). A formulação do conceito de homeostase por Walter Cannon. Filosofia e História da Biologia, 12 (1), $99-113$.

Burford, N. G.; Webster, N. A., \& Cruz-Topete, D. (2017). Hypothalamic-pituitary-adrenal axis modulation of glucocorticoids in the cardiovascular system, International Journal of Molecular Sciences. 18(10). https://doi.org/10.3390/ijms18102150.

Cannon, W. B. (1929). Organization For Physiological Homeostasis. Physiological Reviews, 9, 399-432.

Casarin, S. T., Porto, A. R., Gabatz, R. I. B., Bonow, C. A, Ribeiro, J. P., \& Mota, M. S. (2020). Tipos de revisão de literatura: considerações das editoras do Journal of Nursing and Health. Journal of Nursing and Health.10 (n.esp.), e20104031.

Castro, M., \& Moreira, A. C. (2002). Diagnóstico laboratorial da Síndrome de Cushing. Arquivos Brasileiro de Endocrinologia e Metabologia, 46 (1), 97-105. https://doi.org/10.1590/S0004-27302002000100014.

Castro, M., \& Moreira, A. C. (2003). Análise crítica do cortisol salivar na avaliação do eixo hipotálamo-hipófise-adrenal. Arquivos Brasileiro de Endocrinologia e Metabologia, 47 (4), 358-67. https://doi.org/10.1590/S0004-27302003000400008.

Chernow, B., Alexander, H. R., Smallridge, R. C., Thompson, W. R., Cook, D., Beardsley, D., Fink, M. P., Lake, C. R., \& Fletcher, J. R. (1987). Hormonal Responses to Graded Surgical Stress. Archives of Internal Medicine, 147 (7), 1273-1278.

Cordás, T. A., \& Laranjeiras, M. (2006). Efeitos colaterais dos psicofármacos na esfera sexual. Revista Psiquiatria Clínica, 33 (3), 168-173. https://doi.org/10.1590/S0101-60832006000300007.

Day, T. A. (2005). Defining stress as a prelude to mapping its neurocircuitry: No help from allostasis, Progress in Neuropsychopharmacol Biological Psychiatry, 29 (8), 1195-200. https://doi.org/10.1016/j.pnpbp.2005.08.005

De Rose Jr, D. (2002). A competição como fonte de estresse no esporte. Revista Brasileira de Ciência e Movimento, 10 (4), 19-26. http://dx.doi.org/10.18511/rbcm.v10i4.466.

Farias, E. R.., \& Silva, M. F. L. da. (2021). Mental stress in elite athletes: A systematic review. Research, Society and Development, 10(7), e55710716838. https://doi.org/10.33448/rsd-v10i7.16838.

Faro, A., \& Pereira, M. E. (2013). Estresse: Revisão Narrativa da Evolução Conceitual, Perspectivas Teóricas e Metodológicas. Psicologia, Saúde \& Doenças, $14(1), 78-100$.

Fava, G. A., Mcewen, B. S., Guidi, J., Gostoli, S., Offidani, E., \& Sonino, N. (2019). Clinical characterization of allostatic overload, Psychoneuroendocrinology, 108, 94-101. https://doi.org/10.1016/j.psyneuen.2019.05.028.

Ferreira, R. M., Penna, E. M., Costa, V. T., \& Moraes, L. C. C. A. (2012). Nadadores medalhistas olímpicos: contexto do desenvolvimento brasileiro. Motriz: Revista de Educação Física, 18 (1), 130-142. https://doi.org/10.1590/S1980-65742012000100014.

Freire, C. A., Cuenca, A. L. R., Leite, R. D., Prado, A. C., Rios, L. P., Stakowian, N., \& Sampaio, F. D. F. (2020). Biomarkers of homeostasis, allostasis, and allostatic overload in decapod crustaceans of distinct habitats and osmoregulatory strategies: an empirical approach. Comparative Biochemistry and Physiology -Part A: Molecular and Integrative Physiology, 248. https://doi.org/10.1016/j.cbpa.2020.110750.

Goiato, M. C., da Silva, E., Cândido, N. B., Nóbrega, A. S., de Medeiros, R. A., Sumida, D. H., Chiba, F. Y., \& Dos Santos, D. M. (2019). Avaliação do nível de cortisol, glicemia capilar e pressão arterial em resposta à ansiedade de pacientes reabilitados com próteses totais. BMC oral health, 19 (1), 75. https://doi.org/10.1186/s12903-019-0763-z.

Gallo, L. C., Fortmann, A. L., \& Mattei, J. (2014), Allostatic load and the assessment of cumulative biological risk in biobehavioral medicine: Challenges and opportunities, Psychosomatic Medicine, 76 (7), 478-480. https://doi.org/10.1097/PSY.0000000000000095

Guidi, J., Lucente, M., Sonino, N., \& Fava, G. A. (2020). Allostatic Load and Its Impact on Health: A Systematic Review. Psychotherapy and Psychosomatics, 90 (1), 11-27. https://doi.org/10.1159/000510696.

Inder, W. J., Dimeski, G., RusseL. A. (2012). Measurement of salivary cortisol in laboratory techniques and clinical indications. Clinical Endocrinology, 77 (5), 645-51. https://doi.org/10.1111/j.1365-2265.2012.04508.x.

Kerr, P., Kheloui, S., Rossi, M., Désilets, M., \& Juster, R. P. (2020). Allostatic load and women's brain health: A systematic review. Neuroendocrinology. 59. https://doi.org/ 10.1016/j.yfrne.2020.100858.

Kim, K. J., Chung, J. W., Park, S., \& Shin, J. T. (2009). Psychophysiological stress response during competition between elite and non-elite Korean junior golfers. International Journal of Sports Medicine, 30 (7), 503-8. https://doi.org/ 10.1055/s-0029-1202338.

Kudielka, B. M., Hellhammer, D. H., \& Wüst, S. (2009). Why do we respond so differently? Reviewing determinants of human salivary cortisol responses to challenge. Psychoneuroendocrinology, 34 (1), 2-18. https://doi.org/10.1016/j.psyneuen.2008.10.004.

Liu, B., Zhang, TN, Knight, JK e Goodwin, JE (2019). The Glucocorticoid Receptor in Cardiovascular Health and Disease. Cells, 8 (10), 1227. https://doi.org/10.3390/cells8101227. 
Lightman, S. L., Birnie, M. T., \& Conway-Campbell, B. L. (2020). Dynamics of ACTH and Cortisol Secretion and Implications for Disease. Endocrine reviews, 4 (3), 470-490. https://doi.org/10.1210/endrev/bnaa002.

Loures, D. L., Sant'Anna, I., Baldotto, C. S. R., Sousa, E. B. S., \& Nóbrega, A. C. L. (2002). Estresse Mental e Sistema Cardiovascular. Arquivos Brasileiros de Cardiologia, 78 (5), 525-530. https://doi.org/10.1590/S0066-782X2002000500012.

Maidana, P., Bruno, O. D., \& Mesch. V. (2013). Medición de cortisol y sus fracciones uma puesta al día. Medicina. 73 (6), $579-584$.

Margis, R., Picon, P., Cosner, A. F., \& Silveira, R. O. (2003). Relação entre estressores, estresse e ansiedade. Revista de Psiquiatria do Rio Grande do Sul, 25 (1), 65-47. https://doi.org/10.1590/S0101-81082003000400008.

Matos, S. M. R. de., \& Ferreira, J. C. de S. (2021). Stress and eating behavior. Research, Society and Development, $10(7)$, e26210716726. https://doi.org/10.33448/rsd-v10i7.16726.

Mcewen, B. S. (2016). Stress: Homeostasis, rheostasis, reactive scope, allostasis and allostatic load. In: The Curated Reference Collection in Neuroscience and Biobehavioral Psychology. Elsevier Science Ltd.

Mcewen, B. S. (2000). The neurobiology of stress: From serendipity to clinical relevance. Brain Research, 886(1-2):172-189. https://doi.org/ 10.1016/s00068993(00)02950-4.

Mcewen, B. S., \& Stellar, E. (1993). Stress and the Individual: Mechanisms Leading to Disease. Archives of Internal Medicine, 153(18), 2093-2101. https://doi.org/ 10.1001/archinte.1993.00410180039004.

Mcewen, B. S., \& Wingfield, J. C. (2003). The concept of allostasis in biology and biomedicine. Hormones Behavior, 43 (1), 2-15. https://doi.org/10.1016/S0018-506X(02)00024-7.

Myers, B., Mcklveen, J. M., \& Herman, J. P. (2014). Glucocorticoid actions on synapses, circuits, and behavior: Implications for the energetics of stress, Frontiers in Neuroendocrinology, 35 (2), 180-196. https://doi.org/10.1016/j.yfrne.2013.12.003.

Nicolaides, N. C., Kyratzi, E., Lamprokostopoulou, A., Chrousos, G. P., \& Charmandari, E. (2015). Stress, the stress system and the role of glucocorticoids. NeuroImmunoModulation, 22(1), 6-19. https://doi.org/10.1159/000362736.

Nicolò, A., Massaroni, C., Schena, E., \& Sacchetti, M. (2020). The Importance of Respiratory Rate Monitoring: From Healthcare to Sport and Exercise. Sensors, 20(21), 6396. https://doi.org/10.3390/s20216396.

Pacák, K., Palkovits, M. (2001). Stressor specificity of central neuroendocrine responses: Implications for stress-related disorders, Endocrine Reviews, 22 (4) 502-48. https://doi.org/10.1210/edrv.22.4.0436.

Pereira, M. C., \& Ribeiro, L. (2012). Stresse, Catecolaminas e Risco Cardiovascular. Arquivos de Medicina, 26 (6), $245-253$.

Poletto, M., Koller, S. H., \& Dell'aglio, D. D. (2009). Eventos estressores em crianças e adolescentes em situação de vulnerabilidade social de Porto Alegre. Ciência \& Saúde Coletiva, 14 |(2), 455-66. https://doi.org/10.1590/S1413-81232009000200014.

Ramamoorthy, S., \& Cidlowski, J. A. (2016). Corticosteroids. Mechanisms of Action in Health and Disease, Rheumatic Disease Clinics of North America, 2(1),15-31, https://doi.org/10.1016/j.rdc.2015.08.002.

Ranabir, S., \& Reetu, K. (2011). Stress and hormones. Indian Journal Endocrinology and Metabolism, 15(1):18-22. https://doi.org/10.4103/2230-8210.77573.

Reina-Couto, M., Afonso, J., Carvalho, J., Morgado, L., Ronchi, F. A., Leite, A. P.O., Dias, C. C., Casarini, D, E., Bettencout, P., \& Albino-Teixeira, A. (2020) Interrelationship between renin-angiotensin-aldosterone system and oxidative stress in chronic heart failure patients with or without renal impairment. Biomedicine \& Pharmacotherapy, 113. https://doi.org/10.1016/j.biopha.2020.110938.

Reppert, S. M., \& Weaver, D. R. (2002). Coordination of circadian timing in mammals, Nature, 418, 935-941, 2002. https://doi.org/10.1038/nature00965.

Reynoso-Sánchez, L. F., Flores, J. R. H., García-Dávila, M., Taraco, A. G. R., Jaenes Sánchez, J. C., López-Walle, J. M., Hernández-Cruz, G. (2017). Cortisol y estrés-recuperación durante un periodo competitivo en jugadores de balonmano. Revista de Psicologia del Deporte, 26 (2), $125-131$.

Russell, G. M., Kalafatakis, K., \& Lightman, S. L. (2015). The Importance of Biological Oscillators for Hypothalamic-Pituitary-Adrenal Activity and Tissue Glucocorticoid Response: Coordinating Stress and Neurobehavioural Adaptation, Journal of Neuroendocrinol, 27(6), 378-88. https://doi.org/10.1111/jne.12247.

Schulte, P. M. (2014). What is environmental stress? Insights from fish living in a variable environment. Journal of Experimental Biology, 217 (1), 23-34. https://doi.org/10.1242/jeb.089722.

Selye, H. A. (1936). Syndrome produced by diverse nocuous agents. Nature, 138 (32), 1936.

Selye, H. A. (1959). Stress, a tensão da vida. Editora Ibrasa - Instituição Brasileira de Difusão Cultural.

Shirtcliff, E. A., Buck, R. L., Laughlin, M. J., Hart, T., Cole, C. R., \& Slowey, P. D. (2015). Salivary Cortisol Results Obtainable Within Minutes of Sample Collection Correspond with Traditional Immunoassays. Clinical Therapeutics, 37 (3), 505-14. https://doi.org/10.1016/j.clinthera.2015.02.014.

Shonkoff, J. P., Boyce, W. T., \& Mcewen, B. S. (2009). Neuroscience, molecular biology, and the childhood roots of health disparities: Building a new framework for health promotion and disease prevention. JAMA, 301 (21), 2252-9. https://doi.org/10.1001/jama.2009.754.

Silva, M. L., Mallozi, M. C., \& Ferrari, G. F. (2007). Cortisol salivar na avaliação do eixo hipotálamo-hipofisário-adrenal em crianças saudáveis menores de 3 anos. Jornal de Pediatria, 83 (2), 121-126. https://doi.org/10.1590/S0021-75572007000200005. 
Research, Society and Development, v. 10, n. 17, e82101723561, 2021

(CC BY 4.0) | ISSN 2525-3409 | DOI: http://dx.doi.org/10.33448/rsd-v10i17.23561

Soares, A., \& Pereira, M. (2006). Cortisol como variável em psicologia da saúde. Psicologia, saúde e doenças, 7 (2), 165-177.

Sousa, M. B. C., Silva, H. P. A., \& Galvão-Coelho, N. L. (2015). Resposta ao estresse: I. Homeostase e teoria da alostase. Estudos de Psicologia, 20 (1), $2-11$. https://doi.org/10.5935/1678-4669.20150002.

Sterling, P.; Eyer, J. \& Allostasis: (1988). A New Paradigm to Explain Arousal Pathology. In: Handbook of Life Stress, Cognition and Health. In: Handbook of Life Stress, Cognition and Health: Edited by S. Fisher and J. Reasonp.

Steven J. Forrester, S. J., Booz, G. W., Sigmund, C. D., Coffman, T. M., Kawai, T., Rizzo, V., Scalia, R., \& Eguchi, S. (2018). Angiotensin II Signal Transduction: An Update on Mechanisms of Physiology and Pathophysiology. Physiological Reviews, 98 (8), 1627-1728. https://doi.org/10.1152/physrev.00038.2017.

Tanno, A. P., \& Marcondes, F. K. (2002). Estresse, ciclo reprodutivo e sensibilidade cardíaca às catecolaminas, Revista Brasileira de Ciências Farmacêuticas, 38 (3), 273-289. https://doi.org/10.1590/S1516-93322002000300004.

Tricoli, V. A. C. (2010). O estresse emocional e seus efeitos. In Lipp, Marilda (Org.). O adolescente e seus dilemas: orientação para pais e educadores. Editora Papirus.

Ulrich-Lai, Y. M., \& Herman, J. P. (2009). Neural regulation of endocrine and autonomic stress responses, Nature Reviews Neuroscience, 10 (6), $397-409$. https://doi.org/10.1038/nrn2647.

Vallejo, M. A., Vallejo-Slocker, L., Fernández-Abascal, E. G., \& Mañanes, G. (2018). Determining factors for stress perception assessed with the Perceived Stress Scale (PSS-4) in Spanish and other European samples. Frontiers in Psychology, 26 (9), 1-8. https://doi.org/10.3389/fpsyg.2018.00037.

Veldhuis, J. D., Iranmanesh, A., Lizarralde, G., \& Johnson, M. L. (1989). Amplitude modulation of a burstlike mode of cortisol secretion subserves the circadian glucocorticoid rhythm. American Journal of Physiology, 257 (1), E6-14. https://doi.org/10.1152/ajpendo.1989.257.1.E6.

Verbeeten, K. C., \& Ahmet, A. H. (2018). The role of corticosteroid-binding globulin in the evaluation of adrenal insufficiency, Journal of Pediatric Endocrinology and Metabolism, 31 (2), 107-115. https://doi.org/10.1515/jpem-2017-0270. 